\title{
The Light as Composed of Longitudinal-Extended Elastic Particles Obeying to the Laws of Newtonian Mechanics
}

\author{
Alfredo Bacchieri \\ University of Bologna, Bologna, Italy \\ Email: abacchieri@libero.it \\ Received 16 April 2014; revised 12 May 2014; accepted 7 June 2014 \\ Copyright (C) 2014 by author and Scientific Research Publishing Inc. \\ This work is licensed under the Creative Commons Attribution International License (CC BY). \\ http://creativecommons.org/licenses/by/4.0/ \\ (c) (i) Open Access
}

\begin{abstract}
It is shown that the speed of longitudinal-extended elastic particles, emitted during an emission time $T$ by a source $S$ at speed $u$ (escape speed toward the infinity due to all the masses in space), is invariant for any Observer, under the Newtonian mechanics laws. It is also shown that a cosmological reason implies the light as composed of such particles moving at speed $u$ (function of the total gravitational potential). Compliance of $c$ with Newtonian mechanics is shown for Doppler effect, Harvard tower experiment, gravitational red shift and time dilation, highlighting, for each of these subjects, the differences versus the relativity.
\end{abstract}

\section{Keywords}

Escape Speed, Harvard Tower Experiment, Time Dilation, Redshift, Doppler Effect, Compton Effect

\section{Introduction}

Here we present a solution, in accordance to the Newtonian mechanics, to the apparent constancy of $c$, based on following assumptions:

1) Gravity fields fixed to their related masses (intending that each field is moving together with its generating mass).

2) Finite mass of the universe, implying a finite value of $U$ (total gravitational potential) and therefore of $u$ (escape speed from the universe due to all the masses in space).

3) Light composed of longitudinal-extended elastic particles (as defined on §4) moving at speed $c=u$. This 
equality is supported by a cosmological reason, see §2.

On above bases (including, needless to say, Newton's absolute time and space) we find:

a) The relation between $u$ (total escape speed) and $U$ (total gravitational potential), giving to the speed of light the cosmological reason of its value.

b) On Earth, the variation of $u$, (and therefore of $c$ as per assumption III), due to the variation of $U$ (mainly caused by the variable distance Earth-Sun) is, during one year, $\Delta u(=\Delta c) \leq \pm 0.05 \mathrm{~m} \cdot \mathrm{s}^{-1}$, hence within the accuracy of the measured value of $c$.

c) The invariance of the measure of $c$ for any Reference frame under the Newtonian mechanics laws.

d) The longitudinal, generic and transverse Doppler effect for longitudinal-extended elastic particles, as defined, and their physical characterization.

e) As for the Harvard tower experiment [1]-[3], regarding the variation of frequency (or wavelength) between a source (of gamma rays) and an absorber at different height, our relations give a shift equal to the observed and also predicted by the Relativity. Anyhow, with the source on the base (of the tower) the light arriving to the top has, as for the GR, a lower frequency, whereas on our bases, is the length of our particles which decreases (together with c); on the contrary, with source on the top, GR predicts an increase of the frequency of the light arrived to the base, whereas we show that, during the same path (top-base), is the length of these particles which increases (together with $c$ ), giving a red shift. Moreover, as for the value of the compensating speed source-absorber, (necessary to restore their resonance), we point out that the experiment did not give any clear indication about the effective direction of this speed. Indeed, scope of that experiment was to "establish the validity of the predicted gravitational red shift" [2], hence the only value of this speed was taken in consideration; here, on §6, we show that, on our bases, the effective direction of this speed is contrary in both cases (source on top or base), to the one predicted (but not verified) by the Relativity.

f) As for the gravitational time dilation, on $§ 6$, it is shown that taking a source (of light) in altitude, it yields a negative variation of $c$ as well as a negative variation of the frequency $v$ inducing atomic clocks to run faster; moreover, through our Equation (29) regarding a source circling (around the Earth), we obtain, see (46), the exact variation of the ticking time of GPS system.

g) As for high red shifts related to far sources, we show that, disregarding the relative motion Earth-source, they depend on the increase of $c$ (as well as the increase of the length of the said "longitudinal-extended elastic particles”) during the path of light toward higher (in absolute value) potential; on §7, Table 1, we give the values of $c$ (on these far sources) related to the observed red shifts.

h) Our Equation (17), (regarding our Doppler effect for the light), applied to the Compton effect (indubitable Doppler effect), gives, see Appendix A, the Compton equation, which cannot be obtained through the relativistic Doppler effect equations.

\section{Total Escape Speed (from a Point toward the Infinity) Due to All the Masses in Space}

As known, considering in space one only mass $M$ (regarded as a point-like), the gravitational potential $U$ acting on a particle having mass $m \ll M$, assuming $U_{\infty}=0$, with $s$ the distance $M-m$, is $U=-M G / s$; this relation, according to our first assumption (I), is always valid in spite of any reciprocal motion between $M$ and $m$. The related Conservation of Energy (CoE), $E=U+K$, (where $K=\frac{1}{2} u^{2}$ represents the unitary, i.e. for unit of mass, kinetic energy of our particle arriving from the infinity, where $u_{\infty}=0$ ), for $E=0$ gives $U=-K$, leading to

$$
u=\sqrt{2 K}=\sqrt{-2 U}=\sqrt{2 M G / s}
$$

which is a scalar, (called escape speed), representing (in the considered point) the value of the velocity $\mathbf{u}$, any massive particle, under a potential $U$, needs to reach the infinity, so $\mathbf{u}$ (escape velocity)must be referred to $M$.

Considering now two masses $M_{1}$ and $M_{2}$, having, at a given time, distances $s_{1}$ and $s_{2}$ from a considered point (we may call it Emission point $\mathrm{E}_{\mathrm{p}}$ ), the potential $U_{1,2}$ in $\mathrm{E}_{\mathrm{p}}$ becomes

$$
U_{1,2} \equiv U_{1}+U_{2}=-\left(M_{1} G / s_{1}\right)-\left(M_{2} G / s_{2}\right)
$$

Now, the escape speed from two masses can be written

$$
u_{1,2} \equiv \sqrt{-2 U_{1,2}} \equiv \sqrt{-2\left(U_{1}+U_{2}\right)}=\sqrt{\left(2 M_{1} G / s_{1}\right)+\left(2 M_{2} G / s_{2}\right)}
$$


which is the value, in the considered point $\mathrm{E}_{\mathrm{p}}$ of the (escape) velocity $\mathbf{u}_{1,2}$ which has to be referred (at the considered time), to the point, we may call it Centre of potential $\left(\mathrm{C}_{\mathrm{p}}\right)$, where $\left|U_{1,2}\right|$ has the max value. Then, as $\sqrt{2 M_{1} G / s_{1}}=u_{1}$ and $\sqrt{2 M_{2} G / s_{2}}=u_{2}$ we also get

$$
u_{1,2}^{2}=u_{1}^{2}+u_{2}^{2}
$$

therefore the escape speed due to all the $n$ masses in space becomes

$$
u=\sqrt{\sum u_{n}^{2}}=\sqrt{-2 U}=\sqrt{\sum 2 M_{n} G / s_{n}}
$$

with $\sum M_{n} \equiv M_{\mathrm{u}}$ the universe mass, $U\left(=-\sum M_{n} G / s_{n}=-\frac{1}{2} u^{2}\right)$ the total gravitational potential in the considered point $\mathrm{E}_{\mathrm{p}}$, and where $u$ (function of $U$ in $\mathrm{E}_{\mathrm{p}}$ ) can be called as total escape speed (toward the infinity), while the escape velocity $\mathbf{u}$ is referred to the centre $\mathrm{C}_{\mathrm{p}}$. Indeed, any unitary massive particle during its path toward the infinity, has to comply with the CoE, $U+K=0$, where $K=\frac{1}{2} u^{2}$ giving to this particle a speed $u$ (which depends on the location of the source) and yielding, for all the masses, the total energy equal to zero [Compliance of light with above relation $E=U+K$, is shown on Appendix B].

We assume now the equality $c=u$, hereafter supported by the estimated mass of universe and also by a cosmological reason: in fact, if $c>u$ the energy of light will be lost forever and furthermore the observable masses, following the always increasing mass of light going toward the infinity, will also tend to the infinity moving away from each other. On the contrary, if $c<u$, all the masses in space (having speed lower than $u$ ), will tend to a gravitational collapse, whereas for $c=u$, the mass of light, tending to the infinity in an unlimited time, will avoid the two said events (collapse or dispersion).

Now the mass of universe, by some authors, is estimated [4]-[6] to be $M_{\mathrm{u}} \cong 10^{53} \mathrm{~kg}$; the same order of magnitude is given through the number $\left(\cong 10^{22}\right)$ of observable stars [7] [8], and since from Earth the distribution of the masses appears to be homogeneous and isotropic, under our assumption $U_{\infty}=0$, we may assume their density as decreasing toward the infinity like a function $\rho=\rho_{\mathrm{c}} \mathrm{e}^{-a s}$ with $\rho_{\mathrm{c}} \cong 9.2 \times 10^{-27} \mathrm{~kg} / \mathrm{m}^{3}$ the critical density [9]. So the mass of universe can be written

$$
M_{\mathrm{u}}=\int_{0}^{\infty} 4 \pi \mathrm{s}^{2} \rho_{c} \mathrm{e}^{-a s} \mathrm{~d} s=4 \pi \rho_{c} \int_{0}^{\infty} s^{2} \mathrm{e}^{-a s} \mathrm{~d} s=\frac{8 \pi \rho_{c}}{a^{3}} \cong 10^{53} \mathrm{~kg}
$$

yielding

$$
a=\left(8 \pi \rho_{c} / M_{\mathrm{u}}\right)^{\frac{1}{3}} \cong 1.3 \times 10^{-26} \mathrm{~m}^{-1}
$$

On Earth, the variation of potential due to an increase of the distance ds, can be written as $\mathrm{d} U=-\mathrm{d} m G / \mathrm{s}$ where $\mathrm{d} m=\rho 4 \pi \mathrm{s}^{2} \mathrm{~d} s$ with $\rho=\rho_{\mathrm{c}} \mathrm{e}^{-a s}$, hence the potential on Earth becomes

$$
U_{0}=-\int_{0}^{\infty}\left(4 \pi \mathrm{s}^{2} / \mathrm{s}\right) G \rho_{c} \mathrm{e}^{-a s} \mathrm{~d} s=-4 \pi \rho_{c} G \int_{0}^{\infty} \mathrm{e}^{-a s} s \mathrm{~d} s=-4 \pi \rho_{c} G / a^{2} \cong-4.5 \times 10^{16} \mathrm{~J}
$$

Now, according to (5), on Earth it is

$$
u_{o}=\sqrt{-2 U_{o}} \cong \sqrt{9 \times 10^{16}} \cong 3 \times 10^{8} \mathrm{~m} / \mathrm{s}
$$

Therefore, on Earth, $u_{0}=c_{0}$, so that $\frac{1}{2} c_{o}^{2}=-U_{o}$ and, in general we may argue

$$
c=\sqrt{-2 U}=u
$$

The equality $c=u$, which implies the massiveness of light, means that, along any free path, the speed of light only depends on the value of the potential along that path.

[As for the relation $c_{o}^{2}=-2 U_{o}$, the Harvard tower experiment has shown that the fractional change in energy (of light) is given by $\delta E / E=-g h / c^{2}$, and since the term $g h$ is the variation of potential from the ground to the height $h$, we may guess that $c^{2}$ has to be related to the total gravitational potential, as also shown on §6]. 


\section{Annual Variation, on Earth, of the Total Escape Speed}

On Earth a small variation of the total escape speed $u_{o}$, from (9), can be written as

$$
\Delta u=\Delta U / u_{o}
$$

where $\Delta U$ is the variation of the total potential on Earth, mainly due to the variable distance Earth-Sun.

So considering the eccentricity $e(=0.0167)$ of Earth's orbit around the Sun, between their average distance $d$ $\left(=1.5 \times 10^{11} \mathrm{~m}\right)$ and their shortest distance (Perihelion) $p=(1-e) d$, and with $u_{0}=3 \times 10^{8} \mathrm{~m} \cdot \mathrm{s}^{-1}$, the (11) gives

$$
\Delta u_{e}=-\frac{\Delta U_{S}}{u_{o}}=\frac{\left[\left(\frac{M_{S} G}{p}\right)-\left(\frac{M_{S} G}{d}\right)\right]}{u_{o}}=+0.05 \mathrm{~m} \cdot \mathrm{s}^{-1}
$$

with $\Delta u_{e}$ the variation of $u$ due to Earth's orbit eccentricity, $\Delta U_{S}$ the variation of potential on Earth due to Sun between the two said distances, with $M_{S}$ the mass of Sun. Hence from Aphelion to Perihelion, one should find $\Delta u_{\mathrm{AP}}\left(=\Delta c_{\mathrm{AP}}\right)=+0.10 \mathrm{~m} \cdot \mathrm{s}^{-1}$ and we note that this variation is compatible with the accuracy of the measured value of $c=299792458 \mathrm{~m} \cdot \mathrm{s}^{-1}$. Due to Earth's rotation, there is also a daily variation which, from midnight to noon, is of the order of $\Delta u_{\mathrm{r}} \cong 2 \times 10^{-4} \mathrm{~m} \cdot \mathrm{s}^{-1}$; so, on Earth, $u_{o}$ is practically constant during one year, as it is for the measurements of the speed of light.

\section{Invariance of $c$ for a Particular Particle, Here Defined, and Related Doppler Effect}

Here we show that the Galileo's velocities composition law, (related to point-particles), cannot be correctly applied to a particle, (hereafter called photon), defined as follows:

"Longitudinally-extended, elastic non divisible particle emitted at speed $u$ by a source during an emission time $T$, and moving along one ray (continuous succession of photons), where two consecutive photons cannot be separated along a free path (constraint of non separation)”.

Of course, more photons emitted during an emission time $T$ need an equal number of rays.

Calling front and tail the extremities of a photon, the constraint of non separation implies that, along a ray, any tail corresponds to the front of the next photon.

Referring to Figure 1(a) (where $\mathrm{E}_{\mathrm{p}}$ is the location of $\mathrm{S}$ at $t=0$ and $\mathrm{S}_{\mathrm{T}}$ its location at $t=T$ ), since the escape velocity $\mathbf{c}(=\mathbf{u})$ of an emitted photon $(\mathrm{AB})$ is referred to the Centre of potential $\mathrm{C}_{\mathrm{p}}$, during its emission time $(0 \leq$ $t \leq T$ ), the term $\mathbf{v}_{\mathrm{CpA}}=\mathbf{u}$ should appear as the velocity of its front $(\mathrm{A})$ from $\mathrm{C}_{\mathrm{p}}$.

The source $\mathrm{S}$ may have a velocity $\mathbf{v}_{\mathrm{CpS}}$ from $\mathrm{C}_{\mathrm{p}}$, thus writing $\mathbf{v}_{\mathrm{CpA}}=\mathbf{v}_{\mathrm{CpS}}+\mathbf{v}_{\mathrm{SA}}$ we should find $\mathbf{v}_{\mathrm{SA}}=\mathbf{u}-\mathbf{v}_{\mathrm{CpS}}$; this means that each photon emitted around the source should have a length $\lambda^{\prime}=\left|\mathbf{v}_{\mathrm{SA}} T\right|=\left|\left(\mathbf{u}-\mathbf{v}_{\mathrm{CpS}}\right)\right| T$ depending on $\mathbf{v}_{\mathrm{Cps}}$, but this is contrary to the experience showing that if the source is fixed to its initial Emission point $E_{p}$ (that is the point where $\mathrm{S}$ is located at the start of the emission) the emitted photons, referring to $\mathrm{E}_{\mathrm{p}}$, have equal characteristics. Thus, during the emission of a photon, the velocity of its front, (to comply with these equal characteristics), has to be referred to the initial Emission point $\mathrm{E}_{\mathrm{p}}$, therefore, see Figure $\mathbf{1 ( b )}$, where $\mathrm{E}_{\mathrm{p}}$ is our reference frame, as for the front $\mathrm{A}$, for definition, we have

$$
\mathbf{v}_{\text {EpA }}=\mathbf{u}
$$

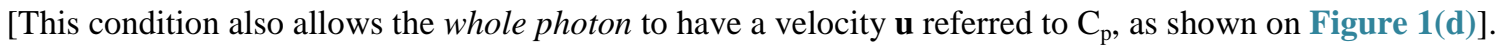

Now the velocity of the front A, with respect to S, from (13), becomes

$$
\mathbf{v}_{\mathrm{SA}}=\mathbf{v}_{\mathrm{SEp}}+\mathbf{v}_{\mathrm{EpA}}=\mathbf{u}-\mathbf{v}_{\mathrm{EpS}}
$$

and still referring to Figure $1(\mathrm{a})$, (where $\mathrm{S}_{\mathrm{T}}$ is the location of $\mathrm{S}$ at $t=T$ ), should $\mathrm{S}$ be fixed to $\mathrm{E}_{\mathrm{p}}$ (that is $\mathbf{v}_{\mathrm{EpS}}=0$ ), the length $\lambda$ of each photon, after the emission time $T$, from (14) becomes $\lambda=v_{\mathrm{SA}} T=u T$, while, in general, it is

$$
\lambda^{\prime}=\mathbf{v}_{\mathrm{SA}} T=\left(\mathbf{u}-\mathbf{v}_{\mathrm{EpS}}\right) T=\lambda-\mathbf{v}_{\mathrm{EpS}} T
$$

where $\lambda^{\prime}$ is the photon $\mathrm{AB}$ emitted with the source in motion from $\mathrm{E}_{\mathrm{p}}$.

Referring now to Figure $\mathbf{1}(\mathrm{c})$, if a generic Observer $\mathrm{O}$ is our Reference frame, we can write 


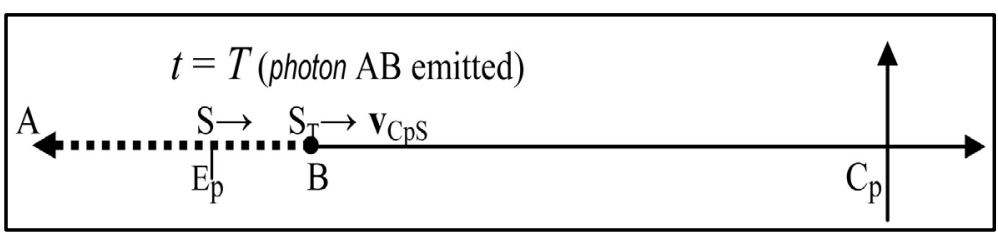

(a)

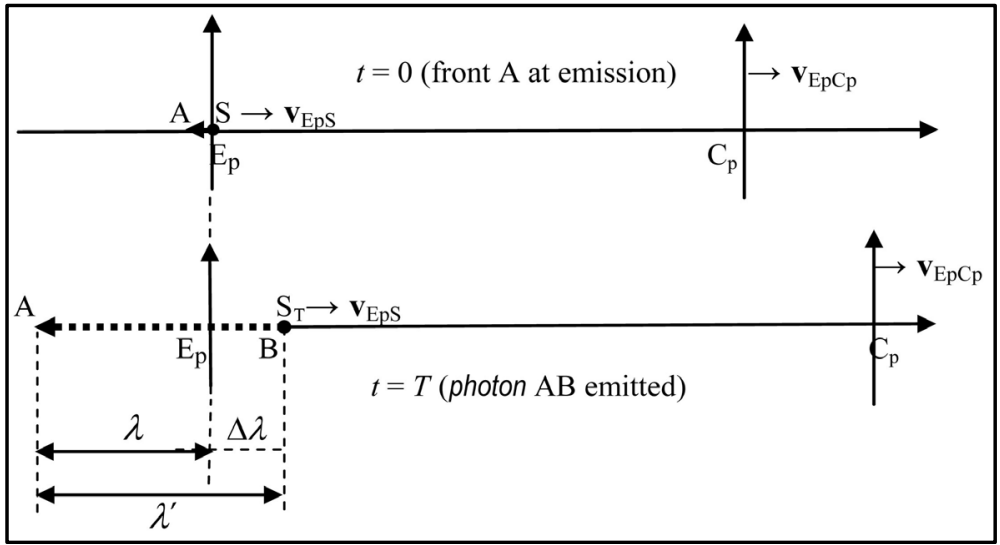

(b)

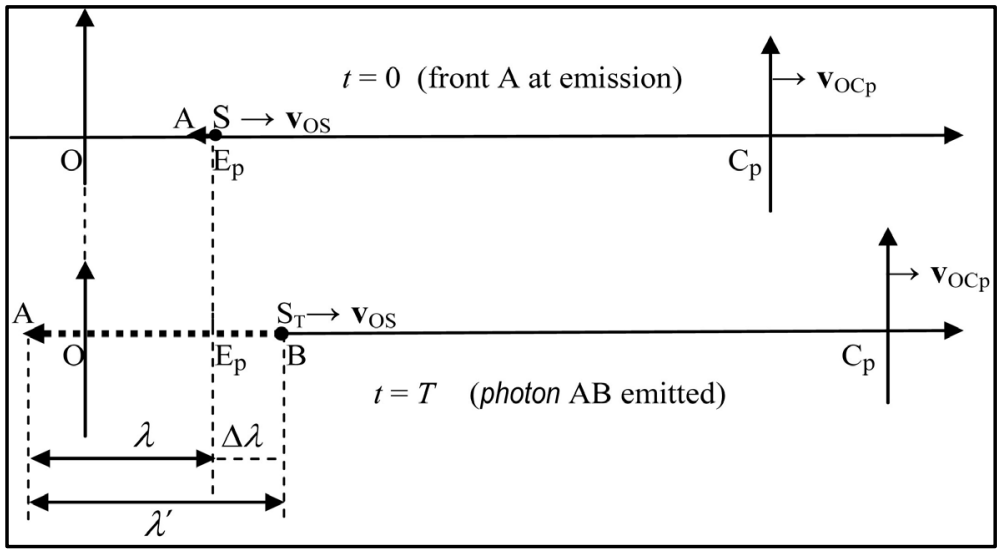

(c)

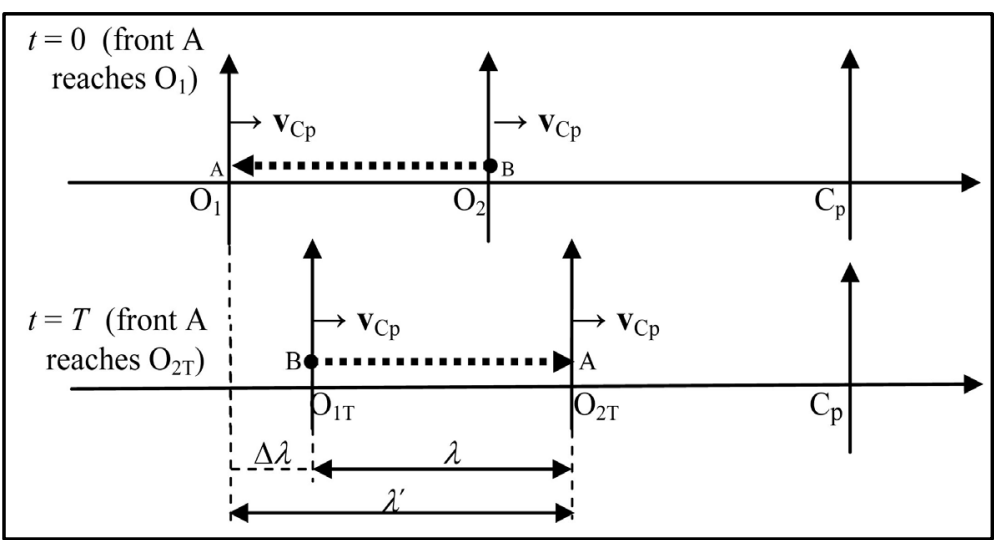

(d)

Figure 1. (a) Photon $\mathrm{AB}$ emitted under the supposed condition $\mathbf{v}_{\mathrm{CA}}=\mathbf{u}$; (b) Emission of a photon $\mathrm{AB}$ referred to the initial Emission point $\mathrm{E}_{\mathrm{D}}$; (c) Emission of a photon $A B$ referred to the generic Observer $\mathrm{O}$; (d) Measurement of the speed of a photon $(\mathrm{AB})$ reflected by $\mathrm{O}_{1}$. 


$$
\lambda^{\prime}=\mathbf{v}_{\mathrm{SA}} T=\left(\mathbf{v}_{\mathrm{SO}}+\mathbf{v}_{\mathrm{OEp}}+\mathbf{v}_{\mathrm{EpA}}\right) T=\left(\mathbf{v}_{\mathrm{SEp}}+\mathbf{u}\right) T=\lambda-\mathbf{v}_{\mathrm{EpS}} T
$$

where $\lambda^{\prime}$ is the photon emitted while the source is in motion, with velocity $\mathbf{v}_{\text {OS }}$, from the Observer, and once more, if $\mathbf{v}_{\mathrm{EpS}}=0$ (S fixed to $\mathrm{E}_{\mathrm{p}}$ ), we find $\lambda^{\prime}=\lambda=u T$ (If $\mathrm{S}$ is now our Reference frame, and $\mathbf{v}_{\mathrm{EpS}}$ is the velocity of $\mathrm{S}$ from $\mathrm{E}_{\mathrm{p}}$, we still have the (15)).

Thus, after the emission time $T$, as for a source receding from the front of the considered photon, as in Figure 1(b) (or Figure 1(c)), the length $\lambda^{\prime}$ (for any Observer) turns out to be

$$
\lambda^{\prime}=u T+v T=\lambda+\Delta \lambda=\lambda(1+\beta)
$$

where $v\left(=\left|\mathbf{v}_{\mathrm{EpS}}\right|\right)$ is the speed (referred to $\left.\mathrm{E}_{\mathrm{p}}\right)$ of the source $\mathrm{S}$ (along the direction $\left.\mathrm{E}_{\mathrm{p}} \mathrm{S}\right), \Delta \lambda(=v T)$ is the path covered by $\mathrm{S}$ during $T$, and where $\beta=v / u$, and we point out that the length $\lambda^{\prime}$ may change, along a free path, and under constant potential, only during its emission.

Now, the speed of a point-particle is defined through two Observers, while the speed $u$ ' of a photon, because of its variable length during its emission, does not correspond to the speed of any point of it, hence we must consider its length referred to the time $T^{\prime}$ (transit time) the photon (front to tail)needs to cross one Observer, so it has to be defined

$$
u^{\prime}=|\lambda-\mathbf{v} T| / T^{\prime}=\lambda^{\prime} / T^{\prime}
$$

[As for this definition, let us consider a system composed of two balls connected through an elastic thread and let them fall in vertical line: during the fall, each part of the system has different speed, so we define the speed of the whole system according to Equation (18)].

Returning now to Figure $1(\mathrm{c})$, for the Observer $\mathrm{O}$, the transit time $T^{\prime}$ of the photon $\mathrm{AB}$ is given by the time the front $\mathrm{A}$ spend to cover the path $\lambda$, that is $T(\lambda / u)$, plus the time the tail B needs to cover the path $S_{T}-E_{P}=\Delta \lambda$; now, once the photon $\mathrm{AB}$ has been emitted (at $t=T$ ), the velocity of the front $\mathrm{A}$ has to be the same as any other part of the emitted photon, hence the time needed by B to cover the path $\Delta \lambda$ is $\Delta T=v T / u$, giving

$$
T^{\prime}=T+\frac{\Delta \lambda}{u}=T+\frac{v T}{u}=T(1+\beta)
$$

Now, according to (18), the speed of the photon AB, referred to O, becomes

$$
u^{\prime}=\frac{\lambda^{\prime}}{T^{\prime}}=\frac{\lambda(1+\beta)}{T(1+\beta)}=u
$$

showing that the speed of photons emitted by a source $\mathrm{S}$ is invariant for any Observer, in spite of any speed of S with respect to the Observer [After the emission, each part of the photon has same velocity u, meaning that, during the emission, it is the velocity of its inner part to vary in order to change its length in the given time $T$ ].

As for an emitted photon, the measurement of $c$ (through the method $d / t$ ) implies its absorption and reflection by an Observer. In this way, the Observer becomes the source of a new photon, with the Observer/Source located in the Emission point $\mathrm{E}_{\mathrm{p}}$, so we may refer to Figure $1(\mathrm{~b})$, with the source fixed in $\mathrm{E}_{\mathrm{p}}$, finding $u^{\prime}=\lambda / T=u$.

[Anyhow, we may obtain the same result $\left(u^{\prime}=\lambda^{\prime} / T^{\prime}=\lambda / T=u\right)$ as follows:

the measurement of $c$ (through the method $d / t$ ) implies two Observers at a constant relative distance $\mathrm{O}_{1} \mathrm{O}_{2}$; on these bases, see Figure $\mathbf{1}(\mathrm{d})$ where $\mathrm{C}_{\mathrm{p}}$ is now our Reference frame, after the reflection of the photon from $\mathrm{O}_{1}$, at $t=T$, the path covered by the front $\mathrm{A}$ to reach $\mathrm{O}_{2 \mathrm{~T}}$ is given by $\mathrm{O}_{1} \mathrm{O}_{2 \mathrm{~T}}$ that is $\lambda^{\prime}=\lambda+\Delta \lambda$ where $\lambda$ is the length of the emitted photon $\mathrm{AB}$ and where $\Delta \lambda=v_{\mathrm{Cp}} T / c$, with $v_{\mathrm{Cp}}$ the speed of our frame $\mathrm{O}_{1} \mathrm{O}_{2}$ with respect to $\mathrm{C}_{\mathrm{p}}$, yielding $\lambda^{\prime}=\lambda(1+\beta)$ where $\beta=v_{\mathrm{Cp}} / c$. The time needed by the front A to cover the distance $\mathrm{O}_{1} \mathrm{O}_{2 \mathrm{~T}}$ is $T^{\prime}=T+\Delta \lambda / c=T(1$ $+\beta$ ), thus the measured speed (referred to the two Observers) becomes $c^{\prime}=\lambda^{\prime} / T^{\prime}=\lambda / T=c$ in spite of any velocity of the co-moving Observers $\mathrm{O}_{1} \mathrm{O}_{2}$ with respect to $\mathrm{C}_{\mathrm{p}}$ (Anyhow, the Observer $\mathrm{O}_{1}$ could state, for the front $\mathrm{A}$, a velocity $\mathbf{v}_{\mathrm{O}_{1} \mathrm{~A}}$ different from $\mathbf{u}$, if he could measure such a speed)].

For any Observer, the frequency of photons of the same ray has to be defined as $v^{\prime}=n / t$ with $n$ the number of photons crossing the Observer during a time $t$; for $t=T^{\prime}$ (transit time of one photon), it is $n=1$, thus $v^{\prime}=1 / T^{\prime}$, so from (19) we get

$$
v^{\prime}=\frac{1}{T^{\prime}}=v /(1+\beta)
$$


showing that for $v=0$, that is $\beta=0$, we have $v^{\prime}=v$, which is also valid if the Observer (O) and the source (S) belongs to different potential: in fact, for $\mathrm{O}$ and $\mathrm{S}$ at reciprocal rest, the number of photons emitted by $\mathrm{S}$ in a unit time has to be equal, in the same time, to the number of them crossing $O$ (like, for instance, the number of balls falling from the top of a tower with respect to an Observer at the tower base), and this implies $v_{\mathrm{s}}=v_{\mathrm{o}}$.

Now, the Figure 1(c), where a source emits a photon while it is in motion from the Observer O, also represents a longitudinal Doppler effect, which, in general, can be written a

$$
\lambda^{\prime}=\lambda(1 \pm \beta) ; \quad v^{\prime}=v /(1 \pm \beta)
$$

with the sign + for S receding from the Observer, while the sign - is for S approaching it.

Hereafter we get our equations regarding both the generic and the transverse Doppler effect, followed by our relations regarding a source (of light) circling around an Observer.

To get a general relation for the Doppler effect, let us consider, see Figure 2(b), referring to the Observer O, a source $\mathrm{S}$, located in $\mathrm{E}_{\mathrm{p}}($ at $t<0)$, at rest with $\mathrm{O}$. During this time let $\mathrm{S}$ emit photons having length $\lambda(=u T)$ and let $\mathrm{E}_{\mathrm{p}} \mathrm{O}=\lambda$. Then, at $t=0$, let $\mathrm{S}$ start to move from $\mathrm{E}_{\mathrm{p}}$ toward $\mathrm{S}_{\mathrm{T}}$ (reached at $t=T$ ), with velocity $\mathbf{v}$ (referred to $\mathrm{O}$ ) along the generic direction a-a. Now, during the path $\mathrm{E}_{\mathrm{p}} \mathrm{S}_{\mathrm{T}}$, let $\mathrm{S}$ emit a photon $\lambda^{\prime}$ toward O. (On Figure 2(a), the small arrow inside the triangle $\mathrm{E}_{\mathrm{p}} \mathrm{OS}_{\mathrm{T}}$ represents the partial $\lambda^{\prime}$ during its emission.) At $t=T$ (end of emission), according to (16) we have $\lambda^{\prime}=\lambda-\mathbf{v} T$, thus the length of $\lambda^{\prime}$, assuming $v \ll u$, so to consider $\mathrm{E}_{\mathrm{p}} \mathrm{O}=\mathrm{NO}$, with $\mathrm{E}_{\mathrm{p}} \mathrm{N}$ $\perp \mathrm{S}_{\mathrm{T}} \mathrm{O}$, becomes

$$
\lambda^{\prime}=\lambda+v T \cos \alpha=\lambda(1+\beta \cos \alpha)
$$

As for the transit time $T^{\prime}$, as before, we can write $T^{\prime}=T+\Delta \lambda / u=T+(v T \cos \alpha) / u$.

which can also be obtained considering that the front of $\lambda^{\prime}$, following the tail of $\lambda$ (thus directed toward $\mathrm{O}$ ), takes a time $T$ to reach $O$ from $E_{p}$, while the tail of $\lambda^{\prime}$, emitted in $S_{T}$, has to cover the path $S_{T} O=S_{T} N+N O$, spending the time $\Delta T=(v T \cos \alpha) / u$ for the path $\mathrm{S}_{\mathrm{T}} \mathrm{N}$, plus the time $T$ for the path $\mathrm{NO}$ (equal to $\mathrm{E}_{\mathrm{p}} \mathrm{O}$ for $v \ll u$ ), giving

$$
T^{\prime}=T+\left(\frac{v T}{u}\right) \cos \alpha=T(1+\beta \cos \alpha)
$$

yielding

$$
v^{\prime}=v /(1+\beta \cos \alpha)
$$

thus $u^{\prime}=\lambda^{\prime} / T^{\prime}=\lambda / T=u \quad$ (For an opposite direction of $\mathrm{S}$ we get $\lambda^{\prime}=\lambda(1-\beta \cos \alpha)$ and $T^{\prime}=T(1-\beta \cos \alpha)$.

[Ray $\mathrm{S}_{2 \mathrm{~T}} \mathrm{FO}$ : referring now to Figure 2(b), if $\mathrm{S}$, between $T$ and $2 T$, is still moving with same velocity $\mathbf{v}$, the emitted photon $\lambda^{\prime \prime}$ will have same length as $\lambda^{\prime}$, thus its front (at $t=2 T$ ) will reach a point $\mathrm{F}$, (corresponding at the same time to the tail of $\lambda^{\prime}$ ), at a distance $\mathrm{FO}=\mathrm{S}_{2 \mathrm{~T}} \mathrm{~N}=v T \cos \alpha$ from O, so the ray Source-Observer, at $t$ $=2 T$, becomes the line $\mathrm{S}_{2 \mathrm{~T}} \mathrm{FO}$ ].

Transverse Doppler effect: referring to Figure 3(a) left part, where $\mathrm{S}_{\mathrm{T}} \mathrm{O}_{\perp} \mathrm{E}_{\mathrm{p}} \mathrm{S}_{\mathrm{T}}$, should $\mathrm{S}$ start to move at $t$ $=0$ from $E_{\mathrm{p}}$ to $\mathrm{S}_{\mathrm{T}}$ while emitting the photon $\lambda^{\prime}$, according to (16) we can write

$$
\lambda^{\prime}=\sqrt{\lambda^{2}-(v T)^{2}}=\lambda \sqrt{1-\beta^{2}} \quad \text { (valid for } \mathrm{S} \text { approaching } \mathrm{O} \text { ) }
$$

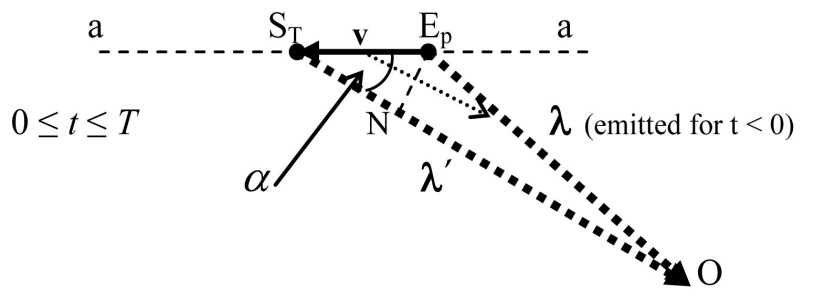

(a)

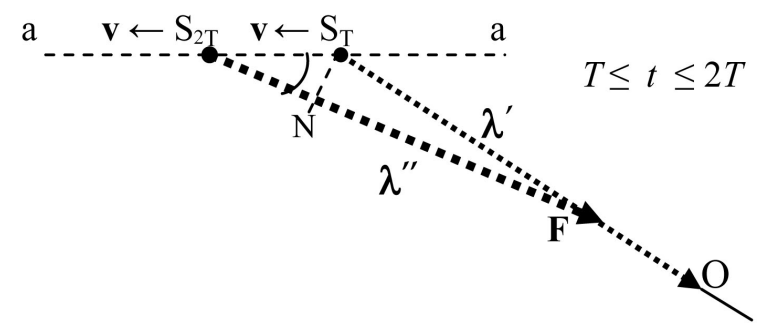

(b)

Figure 2. Doppler effect for a photon, general case. 


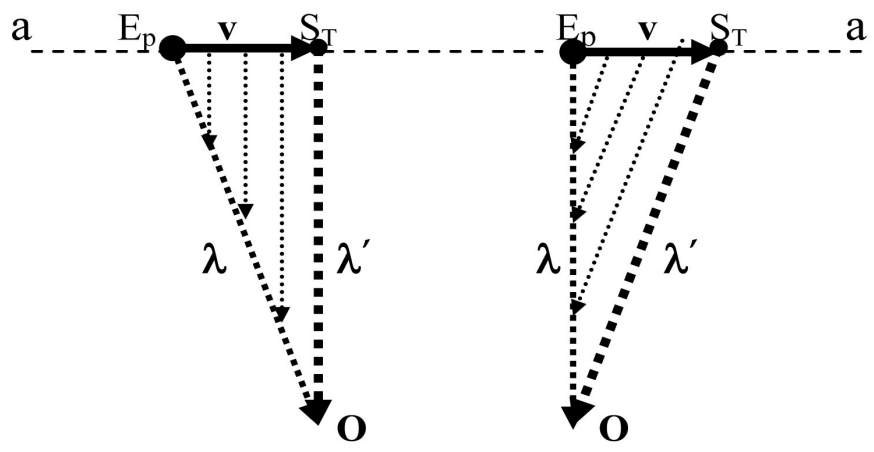

(a)

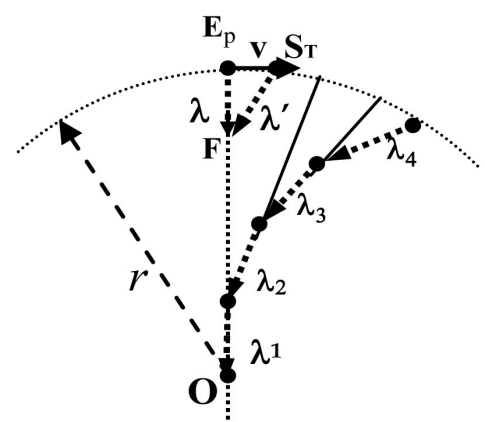

(b)

Figure 3. (a) Transverse Doppler effect; (b) Source circling around the Observer O.

where $\lambda^{\prime}$ is the length corresponding to $\mathrm{S}_{\mathrm{T}} \mathrm{O}$, while $\lambda$ corresponds to $\mathrm{E}_{\mathrm{p}} \mathrm{O}$. On the contrary, see Figure 3(a) right part (where the source is receding from the Observer), it will be

$$
\lambda^{\prime}=\sqrt{\lambda^{2}+(v T)^{2}}=\lambda \sqrt{1+\beta^{2}} \text { (valid for } \mathrm{S} \text { receding from } \mathrm{O} \text { ) }
$$

Then $T^{\prime}=T+\frac{\Delta \lambda}{u}=T+\frac{\lambda^{\prime}-\lambda}{u}=T+\left(\lambda^{\prime} / u\right)-\frac{\lambda}{u}=\lambda^{\prime} / u=\lambda \sqrt{1+\beta^{2}} / u$, hence $c^{\prime}=c$.

Regarding a source circling around an Observer $\mathbf{O}$, on Figure 3(b) the line $\mathrm{E}_{\mathrm{p}} \mathrm{O}$ represents a succession of photons $\lambda$ already emitted when $S$ is fixed in $E_{p}$, while $E_{p} F$ represents the last of them (or it could represent the last photon emitted by $\mathrm{S}$ when reaching $\mathrm{E}_{\mathrm{p}}$ ). Then, at $t=0$ let $\mathrm{S}$ start to move from $\mathrm{E}_{\mathrm{p}}$ with velocity $\mathbf{v}$ toward $\mathrm{S}_{\mathrm{T}}$.

Now, because of the constraint of non separation, the front of the first photon $\lambda^{\prime}$ emitted when $S$ is moving between $E_{p}$ and $S_{T}$, has to reach, in F, the tail of previous photon, so, according to (16) the length of every photon $\lambda^{\prime}$ (emitted while $\mathrm{S}$ is moving along the orbit $r$ ) will be

$$
\lambda^{\prime}=\sqrt{\lambda^{2}+(v T)^{2}}=\lambda \sqrt{1+\beta^{2}}=\lambda \sqrt{1+\omega^{2} r^{2} / c^{2}}
$$

thus

$$
T^{\prime}=T \sqrt{1+\beta^{2}}=T \sqrt{1+\omega^{2} r^{2} / c^{2}}
$$

with $r$ the orbit radius, $\omega$ the angular speed, giving to any whole photon the speed $c^{\prime}=c$.

Figure 3(b) also shows a path $\left(\lambda_{1}-\lambda_{4}\right)$ of a ray directed toward $O$ (the lines connecting the photons $\lambda_{2}$ and $\lambda_{3}$ to the orbit give the point where the source is located at the end of their emission).

\section{Physical Characterization of These Photons}

Now, similarly to a fluid flowing in a pipe (whose kinetic energy is $K=\frac{1}{2} m v^{2}$ with $m$ the mass passing in $1 \mathrm{~s}$ ), the kinetic energy of light flowing along one ray (according to our definition, photons are also massive), has to be expressed with $K_{\mathrm{c}}=\frac{1}{2} m c^{2}$ with $m$ the mass of the particles passing in $1 \mathrm{~s}$ along one ray. Anyhow, the total energy of light flowing along one ray is $E=m c^{2}$ as also proved by the evidences of nuclear reactions like $n+p$ $\rightarrow d+\gamma$ : indeed, in this reaction [10], the lost mass, known through mass spectrometers, corresponds to the value $m=E / c^{2}$ where $E$ ( $=h c / \lambda$ ), (as $\lambda$ is measured), is also known, so $E=m c^{2}$ represents the total energy of light flowing along one ray $\left(\lambda_{\text {meas }}\right.$ is obtained [11] through the value $\lambda_{\text {meas }}\left(\mathrm{d}_{220}\right)$ given at pag. 369 , where $\left(\mathrm{d}_{220}\right)$ is given at page 410).

So, writing $E=\frac{1}{2} m c^{2}+\frac{1}{2} m c^{2}$ we may infer that each of these particles is provided with an internal energy $\left(K_{i}=\frac{1}{2} m c^{2}\right)$ equal to its kinetic energy. Now, equating $m c^{2}$ to $h v$ we get 


$$
m c^{2}=h v(=E)
$$

where $m$ written as

$$
m=\frac{h v}{c^{2}} \equiv \gamma v(\mathrm{~kg})
$$

is the mass of light, with frequency $v$, passing along one ray in $1 \mathrm{~s}$, while the constant

$$
\gamma \equiv h / c^{2}(=m / v)=7.372495 \times 10^{-51} \mathrm{~kg} \cdot \mathrm{s}
$$

is the mass of light passing along one ray during $T$, we may call it "mass of one photon"; so one finds

$$
h=\gamma c^{2}=m c^{2} T
$$

and therefore the Planck's constant represents the energy of one photon. The energy of these particles passing in $1 \mathrm{~s}$ along one ray (energy of one ray of light) can now be written as

$$
E=K_{c}+K_{i}=\frac{1}{2} m c^{2}+\frac{1}{2} m c^{2}=m c^{2}=h v=\gamma c^{2} v
$$

On the above bases, the total energy of light emitted by a source is given by $n_{\mathrm{r}} m c^{2}$ with $n_{\mathrm{r}}$ the number of rays, and since $m$ is the mass of light passing along one ray in $1 \mathrm{~s}$, this unitary (for unit of time) energy shall be equal to the supplied power $P$ during $1 \mathrm{~s}$, thus $n_{\mathrm{r}} m c^{2}=P$, hence the total mass lost per second $m_{\mathrm{T}}\left(\equiv n_{\mathrm{r}} m\right)$ by a source of light becomes

$$
m_{\mathrm{T}}=n_{\mathrm{r}} m=P / c^{2}
$$

So, for a $1 \mathrm{~W}$ lamp, we get $m_{\mathrm{T}}=P / c^{2} \cong 1.1 \times 10^{-17} \mathrm{~kg} \cdot \mathrm{s}^{-1}$, while the number $n_{\mathrm{r}}$ of rays is

$$
n_{\mathrm{r}}\left(=m_{\mathrm{T}} / \gamma v\right)=P / c^{2} \gamma v=P / h v
$$

in our case, $n_{\mathrm{r}} \cong 3 \times 10^{18}$ rays. We point out that for a given power $P$, the higher is the frequency, the lower is the number of rays, as shown by (36) written as $n_{\mathrm{r}} v=P / h$. The number of photons emitted in $1 \mathbf{s}$ becomes:

$$
n_{y}\left(=n_{\mathrm{r}} v\right)=P v / h v=P / h
$$

which, for $P=1 \mathrm{~W}$, gives $n_{\gamma}=h^{-1}\left(=1.5 \times 10^{33}\right.$ photons $\left./ \mathrm{s}\right)$, so the inverse of Planck constant corresponds to the number of photons emitted in $1 \mathrm{~s}$ by a source of unitary power (This great number of photons (having emission time $T$ at speed $c$ ) can be regarded as a wave function).

Now the momentum of the photons passing along one ray in 1s, considering their kinetic energy only, that is $K_{\mathrm{c}}=\frac{1}{2} m c^{2}$, according to Newtonian mechanics should be written as

$$
\mathbf{p}=m \mathbf{c}=\gamma \nu \mathbf{c}=\gamma \mathbf{c} / T
$$

but considering both their kinetic and their internal energy, that is $E=m c^{2}$ we obtain

$$
\mathbf{p}=2 m \mathbf{c}=2 \gamma v \mathbf{c}=2 \gamma \mathbf{c} / T=(\gamma \mathbf{c} / T)+(\gamma \mathbf{c} / T)
$$

\section{Revisitation of the Harvard Tower Experiment and Time Dilation}

Referring to Harvard tower experiment [1]-[3], simply represented on Figure 4, where $h$ is the tower height, calling $c_{0}$ the value of $c$ on Earth's surface at the tower base and $c_{\mathrm{h}}$ its value on its top, the variation $c_{\mathrm{h}}-c_{\mathrm{o}}$ ) from the tower base to its top, from (11), for $c=u$, becomes

$$
\Delta c=c_{h}-c_{\mathrm{o}}=-\Delta U / c_{\mathrm{o}}
$$

where $\Delta U=\left(U_{\mathrm{Eh}}-U_{\mathrm{E0}}\right)$ is the variation of the total gravitational potential $U$, due to Earth, from the tower base to its top. As $U_{\mathrm{Eo}}=-M_{\mathrm{E}} G / r_{\mathrm{o}}$ and $U_{\mathrm{Eh}}=-M_{\mathrm{E}} G / r_{\mathrm{h}}$ where $M_{\mathrm{E}}$ is the Earth's mass and $r$ its radius, we get $\Delta U=M_{\mathrm{E}} G h / r^{2}$ where $h\left(=r_{\mathrm{h}}-r_{\mathrm{o}}\right)$ is the tower height, yielding

$$
\frac{\Delta c}{c_{0}}=\frac{c_{\mathrm{h}}-c_{0}}{c_{\mathrm{o}}}=-\frac{M_{\mathrm{E}} G h}{r^{2} c_{\mathrm{o}}^{2}}=-g h / c_{\mathrm{o}}^{2}
$$

showing that, on the top, where $\left|U_{\mathrm{h}}\right|<\left|U_{\mathrm{o}}\right|$, it is $c_{\mathrm{h}}<c_{\mathrm{o}}$, with $c_{\mathrm{h}}=c_{\mathrm{o}}\left(1-g h / c_{\mathrm{o}}^{2}\right)$. 


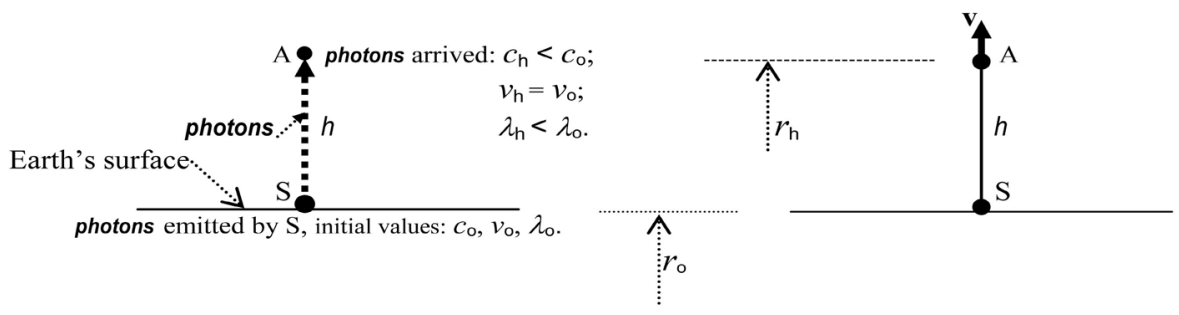

(a)

(b)

Figure 4. Harvard tower experiment scheme, with the source at the base. (a) S and A at rest at a different level $h$. In A, $\lambda_{\mathrm{h}}<\lambda_{\mathrm{o}}$, so the detector observes a gravitational blue-shift; (b) Source and absorber relative motion (v) to compensate the gravitational blue-shift through the Doppler effect.

Now, let $\mathrm{S}$ be a Mossbauer source and A an appropriate absorber; if they are close to each other (for instance, at the tower base), the absorber is in resonance with the source.

Then, see Figure 4(a), with S at the tower base and taking A to its top, while S and A are at rest, the frequency of the emitted photons (i.e. the number of photons emitted along the direction SA per unit of time) has to be equal to the photons received by A, that is $v_{\mathrm{h}}=v_{0}$ and since $c_{\mathrm{h}}<c_{0}$, it must be $\lambda_{\mathrm{h}}<\lambda_{\mathrm{o}}$ (indeed $\Delta \lambda / \lambda_{\mathrm{o}}=\Delta c / c_{0}$ ), so, contrary to ToR, a blue-shift effect for A.

[On Figure 4(a) (photons arrived to the top), according to $v_{\mathrm{h}}=v_{0}$, it seems to be $E_{\mathrm{h}} / E_{\mathrm{o}}=h v_{\mathrm{h}} / h v_{0}=1$ (here $h$ is the Planck constant), but the (33) shows that $h=\gamma c^{2}$ with $\gamma$ (representing the mass of light passing during $T$ along one ray), an effective constant, so that we get $E_{\mathrm{h}} / E_{\mathrm{o}}=\left(c_{\mathrm{h}} / c_{0}\right)^{2}$ which shows a decrease of the energy of light from $\mathrm{S}$ to $\mathrm{A}]$.

Indeed, with $\mathrm{S}$ on the base emitting toward A on top, A goes out of resonance and since on our bases $v_{\mathrm{h}}=v_{0}$, the non-resonances physically related to a variation of $\lambda$, whereas in the Harvard tower experiment [3], "no mention has been made of frequency or wavelength".

Thus, to restore the resonance through the Doppler effect (i.e. to increase the photon length fromits value $\lambda_{\mathrm{h}}$ in A to its initial value $\lambda_{0}$ in S), since $\lambda_{\mathrm{h}}<\lambda_{0}$, A and S, see Figure 4(b), have to recede from each other with speed $v$ complying with (17), here written $\lambda_{\mathrm{o}}=\lambda_{\mathrm{h}}+v T$, giving $\left(\lambda_{\mathrm{h}}-\lambda_{\mathrm{o}}\right) / \lambda_{\mathrm{o}}=-v T / \lambda_{\mathrm{o}}$.

Therefore, since $\Delta \lambda / \lambda_{0}=\Delta c / c_{0}$ (as $v_{0}=v_{\mathrm{h}}$ ), we find $\Delta c / c_{0}=-v T / \lambda_{0}$ and comparing to (41) we get $-v T / \lambda_{\mathrm{o}}=-g h / c_{\mathrm{o}}^{2}$, so the relative speed between $\mathrm{S}$ and $\mathrm{A}$ becomes

$$
v=g h / c_{0}=7.5 \times 10^{-7} \mathrm{~m} / \mathrm{s} \quad(\text { for } h=22.5 \mathrm{~m})
$$

[This value is also predicted by General Relativity (GR)which, implying a decrease of $v$ for light moving from the base to the top, predicts an opposite direction of $\mathbf{v}$ with respect to the one shown on Figure 4(b); at this regard, Pound-Rebka [3] operated in order to determine (through the value of $v$, obtained moving the source sinusoidally) the variation of energy of a beam on the upward and downward path, without any indication (because of the low value of $v$ ), about the direction of the compensating speed].

Now, if we take $\mathrm{S}$ to the tower top, with A located on its base (see Figure 5 which is referred on our bases), the experiment shows that the absorber goes out of resonance.

Now, according to Relativity, taking $S$ to the top, the initial frequency of the light should be $v_{\mathrm{h}}=v_{\mathrm{o}}$, which, on our bases, is wrong: with $\mathrm{S}$ on the top, see Figure 5(a), the (10) written as $c^{2}+2 U=0$, between top and base gives $c_{\mathrm{h}}^{2}+2 U_{\mathrm{h}}=c_{\mathrm{o}}^{2}+2 U_{\mathrm{o}}$, where $U_{\mathrm{h}}-U_{0}=g h$, giving $c_{0}^{2}=c_{\mathrm{h}}^{2}+2 g h$, then $c_{0}=\left(1+2 g h / c_{\mathrm{h}}^{2}\right)^{1 / 2}$, and since $2 g h$ $\ll c^{2}$ we can write $c_{\mathrm{o}}=c_{\mathrm{h}}\left(1+g h / c^{2}\right)$, that is $c_{\mathrm{h}}<c_{\mathrm{o}}$ as showed by (41), but what about $v_{\mathrm{h}}$ and $\lambda_{\mathrm{h}}$ ?

Well, referring to previous Figure 4(a), with source $S$ on the base, the length of photons arriving to the to pvaries from $\lambda_{\mathrm{o}}$ to $\lambda_{\mathrm{h}}$ (with $\lambda_{\mathrm{h}}<\lambda_{\mathrm{o}}$ ), therefore if S has been taken now to the top, should their initial length be $\lambda_{\mathrm{h}}$, at their arrival to the base, their length should be $\lambda_{0}$, and since the resonance, as seen, depends on $\lambda$, the Absorber A (on the base, see Figure 5(a)), should be now in resonance. Thus we can argue that taking the source on top, the photons initial length has to be $\lambda_{\mathrm{h}}\left(=\lambda_{0}\right)$; then, as $c_{\mathrm{h}}<c_{0}$ as shown by (41), it must be $v_{\mathrm{h}}<v_{0}$, and in particu lar, according to (41) we get $\Delta c / c_{\mathrm{o}}=\Delta v / v_{\mathrm{o}}=-g h / c_{\mathrm{o}}^{2}$, giving $v_{\mathrm{o}}=v_{\mathrm{h}}\left(1+g h / c_{\mathrm{o}}^{2}\right)$ which is the same as the one predicted by GR, but on our bases, this variation is due to a different initial frequency (as the source is now on the top), whereas for GR this variation is related to the path of the emitted light between two different levels. (Indeed on our bases the frequency remains constant during any path, should source and observer be at recipro- 


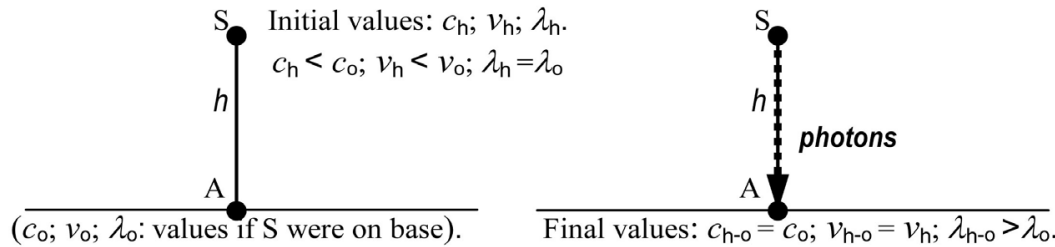

(a)

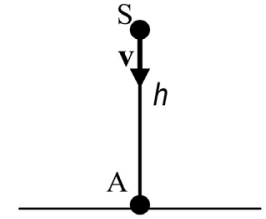

(c)

Figure 5. Harvard tower experiment scheme, with the source on the top. (a) S on top, $\mathrm{S}$ and $\mathrm{A}$ at rest: $c_{\mathrm{h}}, v_{\mathrm{h}}, \lambda_{\mathrm{h}}$ are the photons initial parameters on the tower top; (b) S and A at rest. When photons reach the base, $\lambda_{\mathrm{h}-\mathrm{o}}>\lambda_{0}$, so A observes a g-redshift; (c) S and A relative motion to compensate the g-redshift via Doppler effect.

cal rest).

Thus, see Figure 5(b), with S emitting from the top, S and A at rest, when the photons reach the base, as their final frequency $\left(v_{\mathrm{h}-0}\right)$ will remain the same as the initial one (that is $v_{\mathrm{h}-\mathrm{o}}=v_{\mathrm{h}}$ ) and since $c_{\mathrm{o}}>c_{\mathrm{h}}$, it turns out that, along the path SA, $\lambda$ will increase, and its variation, opposite to the one given by (41), yields now

$\Delta \lambda / \lambda_{\mathrm{o}}=g h / c_{\mathrm{o}}^{2}$, giving $\lambda_{\mathrm{h}-\mathrm{o}}=\lambda_{\mathrm{o}}\left(1+g h / c_{\mathrm{o}}^{2}\right)$.

Now, as $\lambda_{\mathrm{h}-\mathrm{o}}>\lambda_{\mathrm{o}}$, the absorber, on the base, will observe a gravitational red-shift so, to compensate it via Doppler shift, see Figure 5(c), S and A have now to move relative to each other in order to decrease the final length $\lambda_{\mathrm{h}-\mathrm{o}}$ to the resonance value $\lambda_{\mathrm{o}}$; on the contrary, according to ToR, A and S should recede from each other.

[Still referring to Figure 5, taking $\mathrm{S}$ to the tower top, we have $c_{\mathrm{h}}<c_{\mathrm{o}}$ and $v_{\mathrm{h}}<v_{\mathrm{o}}$ implying, contrary to GR, a decrease of the energy of light to be emitted by S, $\left(E_{\mathrm{h}}=h v_{\mathrm{h}}=\gamma c_{\mathrm{h}}^{2} v_{\mathrm{h}}\right)$, see (34), and therefore when these photons reach the base (where $c_{\mathrm{h}-\mathrm{o}}=c_{\mathrm{o}}$ ) their energy becomes $E_{\mathrm{h}-\mathrm{o}}=\gamma c_{\mathrm{o}}^{2} v_{\mathrm{h}}$ giving a reason to the loss of energy as for light arriving to Earth coming from sources located in points where $\left|U_{\mathrm{S}}\right|<\left|U_{\mathrm{o}}\right|$, and since, as seen, $\lambda_{\mathrm{h}-\mathrm{o}}=\lambda_{\mathrm{o}}\left(1+g h / c_{\mathrm{o}}^{2}\right)$, we also give a cosmological reason to the high redshift of sources where $\left|U_{\mathrm{S}}\right| \ll\left|U_{\mathrm{o}}\right|$.

\section{Time Dilation}

Well, the experience shows that, on board of GPS satellites, the atomic clocks run faster by about $38 \mu$ s/day than the ones on ground, meaning that, in altitude, their ticking time, (or interval time, intending the minimum time counted), is shorter than the one on ground.

Now, the ticking time $t$ of atomic clocks is proportional to their frequency, so on ground we can write $t_{0}=k v_{0}$ while in altitude $t_{\mathrm{h}}=k v_{\mathrm{h}}$ yielding $\Delta v / v_{0}=\Delta t / t_{0}$ where $\Delta t\left(=t_{\mathrm{h}}-t_{0}\right)$ is the ticking time variation from ground to height $h$, with $\Delta v\left(=v_{\mathrm{h}}-v_{0}\right)$ representing their frequency variation due to the gravitational potential variation.

Now, taking the sources (clocks) from ground to height $h$, the length of their photons, at emission, remains constant, $\left(\lambda_{\mathrm{h}}=\lambda_{\mathrm{o}}\right)$, thus, because of the variation of $c$ (from ground to height $h$ ), it has to correspond an equal variation of $v$, so that the (40) can be written as

$$
\Delta c / c_{\mathrm{o}}=\Delta v / v_{\mathrm{o}}=\Delta t / t_{\mathrm{o}}=-\Delta U_{E} / c_{\mathrm{o}}^{2}
$$

Now, GPS satellites have an orbit of $r_{\mathrm{h}} 26,600 \mathrm{~km}$, that is an altitude $h 20,200 \mathrm{~km}$, as $r_{\mathrm{o}} 6400 \mathrm{~km}$ is the Earth's radius. Hence, the (43), because of the variation of the potential, the variation of the counted time during one day $\left(\Delta t_{1 d}\right)$, since in one day $t_{1 d}=86,400 \mathrm{~s}$, gives

$$
\Delta t_{1 d}=-\left(\frac{\Delta U_{E}}{c_{0}^{2}}\right) t_{1 d}=-\left[\frac{M_{E} G\left(r_{h}-r_{\mathrm{o}}\right)}{r_{h} r_{0} c_{0}^{2}}\right] 86,400=-45.6 \mu \mathrm{s}
$$

where the sign means that the ticking time is decreasing, inducing the clocks to run faster. Then we have to take into account that the parameters of the photons emitted by atomic clocks on board of GPS satellites are changing because they are circling around the Earth.

Therefore, according to (29), that is $T^{\prime}=T\left(1+\beta^{2}\right)^{1 / 2}$ where $T^{\prime}$ is the time a photon needs to cross the Observer, during one day $(86,400 \mathrm{~s})$, since the orbital speed corresponds to two orbits every day (giving $v=2\left(2 \pi r_{\mathrm{h}} / 86,400\right.$ ) 
$=3870 \mathrm{~m} \cdot \mathrm{s}^{-1}$, and considering that for $v \ll c$ we can write $\left(1+\beta^{2}\right)^{1 / 2} \cong 1+\beta^{2} / 2$, we get

$$
\Delta t_{1 d}^{\prime}=\left(T^{\prime}-T\right) 86,400=\left(\beta^{2} / 2\right) 86,400=7.2 \mu \mathrm{s}
$$

representing the variation of the counted time in one day due to the orbital speed of GPS satellite, and since this variation is positive, it has to be deducted from the negative one due to the potential variation, thus the total variation of the counted time on GPS satellites, in one day, becomes

$$
\Delta t_{\text {day }}=\Delta t_{1 d}+\Delta t_{1 d}^{\prime}=-38.4 \mu \mathrm{s} / \text { day }
$$

as observed. This equality also confirms that $\lambda_{\mathrm{h}}=\lambda_{\mathrm{o}}$ as for sources in altitude.

\section{Red Shift}

According to the Relativity, the gravitational red shift of light coming from the Sun, with $M_{\mathrm{S}}$ and $R_{\mathrm{S}}$ its mass and radius, is $z_{\mathrm{S}} \cong M_{\mathrm{S}} G / R_{\mathrm{S}} c^{2}=2 \times 10^{-6}$. Now, for $s<20 \mathrm{Mpc}$, with $s$ the distance Earth-source, the observed shifts are in the range $z \cong \pm 10^{-2}$ [12], while from $\cong 20$ to $\cong 40$ Mpc they tend to become always positive, and between $\cong 40$ and $\cong 900 \mathrm{Mpc}(\cong 3$ Bly) the red shifts (here in the range $\cong 0.01-0.20$ ), practically follow the empirical Hubble's law $z=H_{0} \mathrm{~s} / \mathrm{c}$; hence, since the value of the gravitational red shifts of a typical galaxy, intended to be $z_{\mathrm{g}} \cong M_{g} G / R_{g} c^{2}$, should be of the order of $10^{-9}$, the Doppler effect appears to be (as for the Relativity), the only satisfactory way to explain the observed blue shifts and also the high (cosmological) red shifts.

On the contrary, on our basis, disregarding any motion between a source and an Observer on Earth, which implies (as showed on §4) $v=v_{0}$, we get $c / \lambda=c_{0} / \lambda_{0}$, where $v_{0}, c_{0}$ and $\lambda_{0}$ are observed on Earth, showing that for $c_{0}>c$, it has to be $\lambda_{0}>\lambda$. Hence, the blue/red shifts observed on Earth can be expressed as

$$
z \equiv \Delta \lambda / \lambda=\Delta c / c=\left(c_{0}-c\right) / c=\left(c_{0} / c\right)-1=\sqrt{U_{0} / U_{s}}-1
$$

where $U_{0}\left(=-\frac{1}{2} c_{0}^{2}\right)$ is the potential on Earth, while $U_{s}$ the one on the source (at distance $s$ ). Thus the shift of a far source, disregarding the motion source-Earth, turns out to be the variation of $c$ (as well as $\lambda$ ) during the path of light toward a different potential; for instance, going from Earth to Sun, and considering that along this path the main variation of potential $\left(\Delta U_{(\mathrm{s})}\right)$ is due to the Sun only, we can write $U_{\mathrm{S}}=U_{\mathrm{o}}+\Delta U_{(\mathrm{s})}$ where

$$
\Delta U_{(s)}=-\left(M_{s} G / R_{s}\right)+\left(M_{s} G / d\right) \cong-M_{s} G / R_{s}
$$

With $d$ the distance Earth-Sun; so on the Sun, $\quad c_{\mathrm{s}}=\sqrt{-2 U_{\mathrm{s}}}=\sqrt{-2\left(U_{\mathrm{o}}+\Delta U_{(\mathrm{s})}\right)}$ and then

$$
c_{s}=\sqrt{c_{0}^{2}+2 M_{s} G / R_{s}} \cong c_{\mathrm{o}}\left(1+\frac{M_{s} G}{R_{s} c_{0}^{2}}\right)=c_{\mathrm{o}}+635 \mathrm{~m} \cdot \mathrm{s}^{-1}
$$

giving $\Delta c / c=z \cong 2.1 \times 10^{-6}$, while on the opposite path, Sun to Earth, it is $z \cong 2.1 \times 10^{-6}$, hence a blueshift (contrary to a red shift of the same value predicted by the Relativity).

As for $s<\cong 40 \mathrm{Mpc}$, according to (47), if $U_{s}$ (potential on the source) is, in absolute value, higher than the potential on Earth $U_{0}$, we get, on Earth, $z<0$ (blue shift), and vice versa for $\left|U_{s}\right|<\left|U_{\mathrm{o}}\right|$, hence, apart Doppler effects, these red/blue shifts indicate that the potential, from Earth to the sources in this space, may increase or decrease (and since for $s>\cong 40 \mathrm{Mpc}, z$ is positive, we may also argue that our galaxy is close to the middle of the masses of universe); then, over this distance, it turns out that, on the related sources, $U_{S}$ is (in absolute value), always lower than $U_{0}$, and also tending to zero for $z \rightarrow \infty$.

In the range $\cong 0.01<z<\cong 0.20$ (where $z$ follows the Hubble's law), the (47), written as

$$
U_{s}=U_{0} /(1+z)^{2}
$$

for $z \ll 1$ gives $U_{s} \cong U_{\mathrm{o}} /(1+2 z)$ yielding (through a simple artifice) to

$$
U_{s} \cong U_{\mathrm{o}}(1-2 z) \quad(\text { valid for } z \ll 1)
$$

which shows that, for $z \ll 1, U_{s}$ depends linearly on $z$; in particular, Table 1 shows that, in the said range 
Table 1. Calculated values of $U$ and $c$ related to the observed redshifts on Earth. The $4^{\text {th }}$ column is referred to Equation (50); the $5^{\text {th }}$ to (51); the $6^{\text {th }}$ to (47).

\begin{tabular}{|c|c|c|c|c|c|}
\hline Redshift $z$ & $s=z c / H_{0} M p c$ & $\begin{array}{c}1 \mathrm{Mpc} 3.3 \times 10^{6} \text { ly } \\
\text { light years }\end{array}$ & $U_{s} / U_{0}=1 /(z+1)^{2}$ & $U_{s} / U_{0}=(1-2 z)$ & $c_{s} / c_{0}=1 /(z+1)$ \\
\hline$<|0.01|$ & $<43$ & $<140$ Mly & $0.98-1.02$ & $0.98-1.02$ & $0.99-1.01$ \\
\hline 0.01 & 43 & 140 Mly & 0.98 & 0.98 & 0.99 \\
\hline 0.05 & 215 & 700 Mly & 0.90 & 0.90 & 0.95 \\
\hline 0.20 & 860 & 2.8 Bly & 0.69 & 0.60 & 0.83 \\
\hline 1.0 & & & 0.25 & & 0.50 \\
\hline 5.0 & & & 0.028 & & 0.17 \\
\hline 9.0 & & & 0.010 & & 0.10 \\
\hline
\end{tabular}

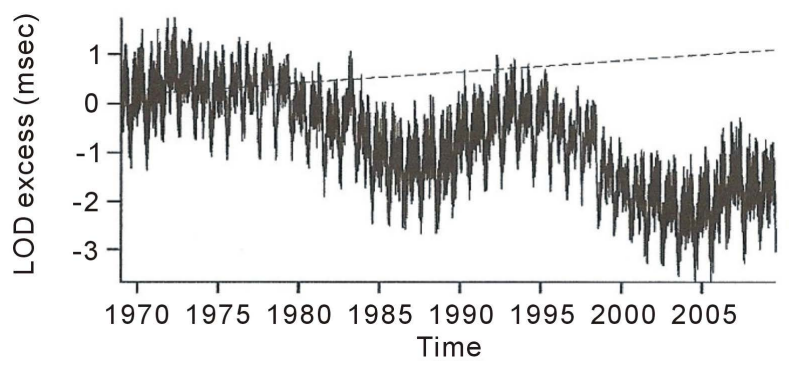

Figure 6. Length of day since 1969, when Earth-Moon laser ranging data started to be collected. The dotted line shows the $2.3 \mathrm{msec}^{-\mathrm{cy}^{-1}}$ trend expected as a consequence of momentum conservation, when it is assumed that, using laser ranging, an actual increase of Earth-Moon distance is measured. LOD data comes from the EOP 05. C04 series (Bizouard and Gambis 2009), as provided by the Earth Orientation Centre (http://hpiers.obspm.fr). The Figure has been taken from the paper of Sanejouand, Y.H., Empirical evidences in favour of avarying-speed-of-light, arXiv 0908.0249.

( $\cong 0.01-0.20$ ), the values given by (51), are practically the same as the ones given by (50). Table 1 also shows the values of $U$ and $c$ for various values of $z$.

\section{Conclusions}

We showed that, on our basis, $c$ corresponds to the total escape speed $u$ which is practically constant on Earth (see §3), while the annual variations of $c$, due to the eccentricity of the Earth's orbit, are well shown on following Figure 6.

In fact, when the Earth (on perihelion) approaches the Sun, because of the distortion of the shape of Earth, the angular speed of Earth should decrease, hence, the length of day (LOD)should increase; moreover, during the approach Earth-Sun, due to the increase of the absolute value (on Earth) of $U$, the speed of light (on Earth) has also to increase, thus the LOD and $c$ should show, at the same time, annual peaks, as shown on Figure 1, which may also represent, in another scale, the annual variations of the speed of light, since 1969.

Now we point out our attention on Compton effect: indeed, through the relativistic Doppler effect equations, one cannot get the Compton equation (which can be found in other ways), whereas through our Doppler effect Equation (17), we can obtain it; well, one can observe that the Compton effect is not a Doppler effect, but in this case why we get the Compton equation? Is it a coincidence or the relativistic Doppler effect equations are not correct?

Finally, regarding the Harvard tower experiment, the Relativity, as for the compensating speed source-Observer (in order to restore the resonance between them), predicts opposite directions with respect to the ones we have obtained: we hope that now (after 50 years from the related experiment) an appropriate (similar) experi- 
ment will give a sure answer.

\section{References}

[1] Pound, R.V. and Rebka Jr., G.A. (1960) Physical Review Letters, 4, 337. http://dx.doi.org/10.1103/PhysRevLett.4.337

[2] Pound, R.V. and Snider, J.L. (1964) Physical Review Letters, 13, 539. http://dx.doi.org/10.1103/PhysRevLett.13.539

[3] Pound, R.V. and Snider, J.L. (1965) Physical Review, 140, B788. http://dx.doi.org/10.1103/PhysRev.140.B788

[4] Kragh, H. (1996) Cosmology and Controversy. Princeton University Press, Princeton, 212.

[5] Gogberashvili, M., et al. (2012) Cosmological Parameter. ArXiv:physics.gen-ph, 2.

[6] Immerman, N. (2001) Nat'l Solar Observatory: The Universe. University of Massachusetts, Sunspot.

[7] Gott III, J.R., et al. (2005) The Astrophysics Journal, 624, 463-484.

[8] Van Dokkum, P. (2010) Nature, 468, 940-942. http://dx.doi.org/10.1038/nature09578

[9] Schutz, B.F. (2003) Gravity from the Ground Up. Cambridge University Press, Cambridge, 361. http://dx.doi.org/10.1017/CBO9780511807800

[10] Halliday, D. and Resnic, R. (1981) Fundamental of Physics. John Wiley \& Sons, New York.

[11] Mohr, P.J. and Taylor, B.N. (2000) Review of Modern Physics, 72, 351-495.

[12] (Yearly) NASA Extragalactic Database. 


\section{Appendix A (Compton Effect)}

Here, see Figure A1, an incident photon (length $\lambda$, frequency $v$ ), ejects a circling electron $\left(m_{\mathrm{e}}\right)$ but there is also a reflected photon (length $\lambda^{\prime}$ frequency $v^{\prime}$ ) so the electron, while emitting a photon $\lambda^{\prime}$ toward the Observer A, represents a source in motion from A along the direction $\mathbf{w}$, thus (considering the component $\mathbf{w}_{\mathrm{A}}$ of $\mathbf{w}$ ) there is an indubitable Doppler effect.

Now, on the basis that the scattered photon starts to be reflected at the same time when the incident photon starts to hit the electron, and since $T^{\prime}\left(=1 / v^{\prime}\right)$ is the emission time of the photon $v^{\prime}$, it turns out that $T^{\prime}$ is also the whole interaction time, meaning that there is not a complete absorption of the incident photon followed by an emission: this means that the internal energy of the photon is not involved in this action, hence the momentum transferred from the incident light to the electron is $\mathbf{p}=m \mathbf{c}(=\gamma \mathbf{c} / T)$ as per (38), and the same value $\mathbf{p}=$ $m \mathrm{c}$ is the momentum transferred from the scattered photon to the electron.

Therefore, the Conservation of Momentum (CoM) along the direction normal to $\mathbf{w}$, becomes

$\left(\gamma c / T^{\prime}\right) \sin \theta=\left(\gamma c / T^{\prime}\right) \sin \theta^{\prime}$ giving $\theta=\theta^{\prime}$.

Moreover, the length of the reflected photon, for the Observer, according to (17) is

$$
\lambda^{\prime}=\lambda+\Delta \lambda=\lambda(1+\beta)
$$

where $\Delta \lambda=w_{\mathrm{A}} T^{\prime}$ and where $w_{\mathrm{A}}=w \cos \theta$ is the component of the electron speed along the direction of the Observer A and $T^{\prime}\left(=1 / v^{\prime}\right)$ is, for $\mathrm{A}$, the photon transit time, so we get

$$
\lambda^{\prime}-\lambda(\cong \Delta \lambda)=w T^{\prime} \cos \theta .
$$

Now the CoM along $\mathbf{w}$ is $\left(\gamma c / T^{\prime}\right) \cos \theta+\left(\gamma c / T^{\prime}\right) \cos \theta=m_{\mathrm{e}} w$ giving

$$
w T^{\prime}=(2 \gamma c \cos \theta) / m_{\mathrm{e}}
$$

and plugging this value into (53) we get

$$
\lambda^{\prime}-\lambda(\cong \Delta \lambda)=2 \gamma c \cos ^{2} \theta / m_{\mathrm{e}}=2 h \cos ^{2} \theta / c m_{\mathrm{e}} .
$$

Now, $2 \theta+\varphi=\pi, \Rightarrow \theta=(\pi-\varphi) / 2$, hence $\cos \theta=\sin \varphi / 2$ and therefore

$$
\Delta \lambda=2 h \sin ^{2}(\varphi / 2) / \mathrm{cm}_{\mathrm{e}}
$$

and since $2 \sin ^{2}(\varphi / 2)=(1-\cos \varphi)$, we get the Compton equation:

$$
\Delta \lambda=\lambda^{\prime}-\lambda=h(1-\cos \varphi) / m_{\mathrm{e}} c,
$$

which cannot be obtained through the relativistic Doppler effect equation which, as for a source receding from the Observer, is $\lambda^{\prime}=\lambda\left(1-\beta^{2}\right)^{1 / 2} /(1-\beta)$.

$\varphi$ : angle between the direction of the incident photon and the scattered one $\left(\lambda^{\prime}\right)$

$\theta$ : angle between the direction of the incident photon and the recoiled electron

$\theta^{\prime}:(=\pi-\varphi-\theta):$ it is shown that $\theta^{\prime}=\theta$.

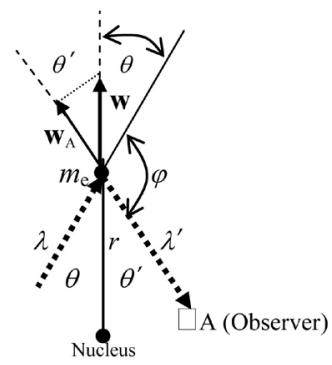




\section{Appendix B}

Regarding the Conservation of Energy (CoE), $E=U+K=0$, leading to $U=-\frac{1}{2} u^{2}$ the term $K$ is the unitary (per unit of mass) kinetic energy of a massive particle, hence as for the light (having total energy, see (34), $E=K_{\mathrm{c}}+K_{\mathrm{i}}=\frac{1}{2} m c^{2}+\frac{1}{2} m c^{2}$ with $K_{\mathrm{i}}$ its internal energy), we have to consider, to comply the CoE, its kinetic energy only, giving $U=-\frac{1}{2} c^{2}$, like any other massive particle. Anyhow, one can observe that the internal energy of light ( $K_{\mathrm{i}}=\frac{1}{2} m c^{2}$ ) of photons going toward the infinity could be lost, but this is not the case: indeed, we have seen on $\S 6$ (see also Figure 4(a)) that the frequency of a photon is constant along its path, and since, see (34), $K_{\mathrm{i}}=\frac{1}{2} m c^{2}=\frac{1}{2} \gamma v c^{2}=\frac{1}{2} \gamma \lambda^{2} v^{3}$, it turns out that toward the infinity, where $c_{\infty} \rightarrow 0$, we get $\lambda_{\infty} \rightarrow 0$, yielding $K_{\mathrm{i} \infty} \rightarrow 0$ (In other words, the internal energy of light only depends on the length of their photons). 
Scientific Research Publishing (SCIRP) is one of the largest Open Access journal publishers. It is currently publishing more than 200 open access, online, peer-reviewed journals covering a wide range of academic disciplines. SCIRP serves the worldwide academic communities and contributes to the progress and application of science with its publication.

Other selected journals from SCIRP are listed as below. Submit your manuscript to us via either submit@scirp.org or Online Submission Portal.
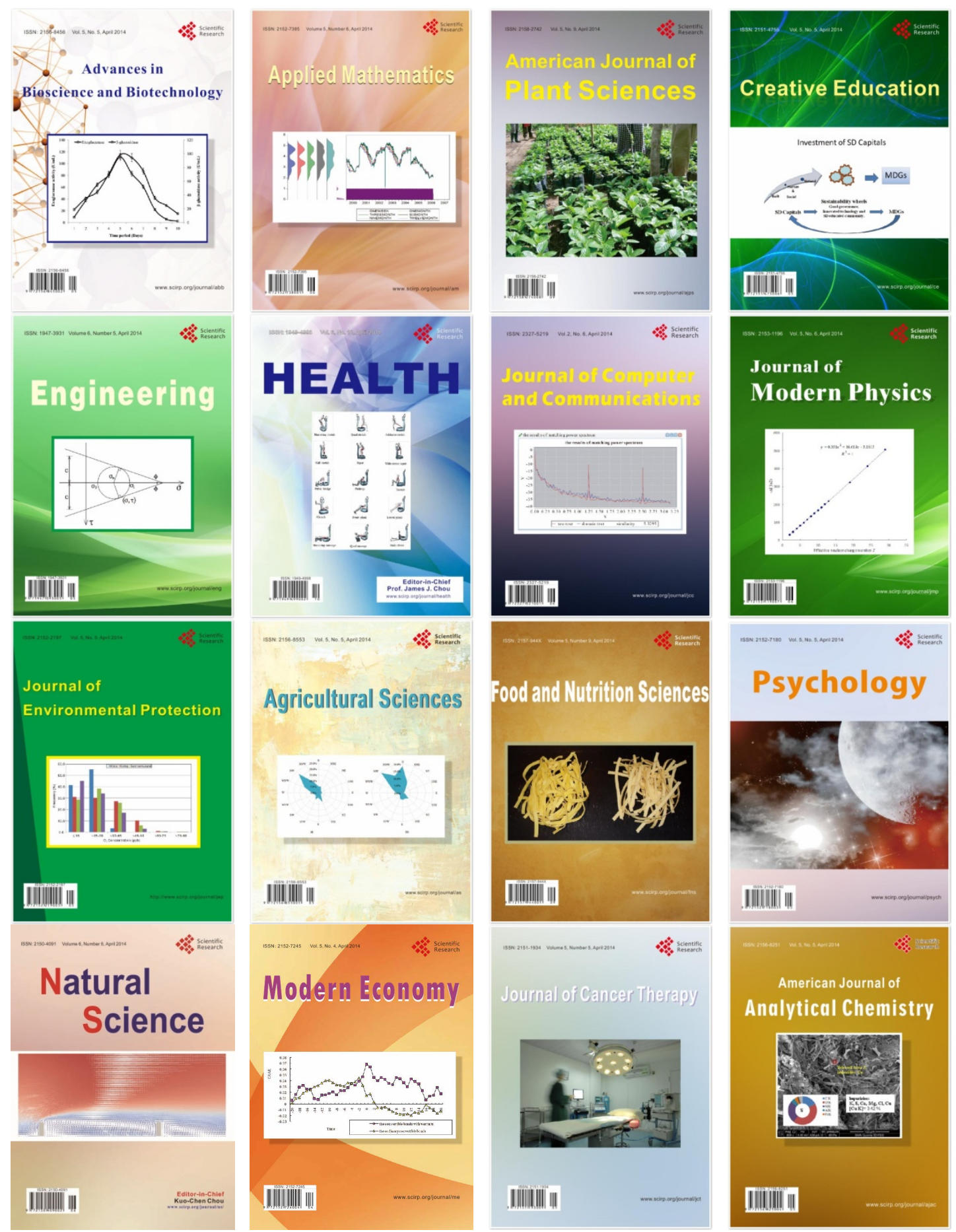